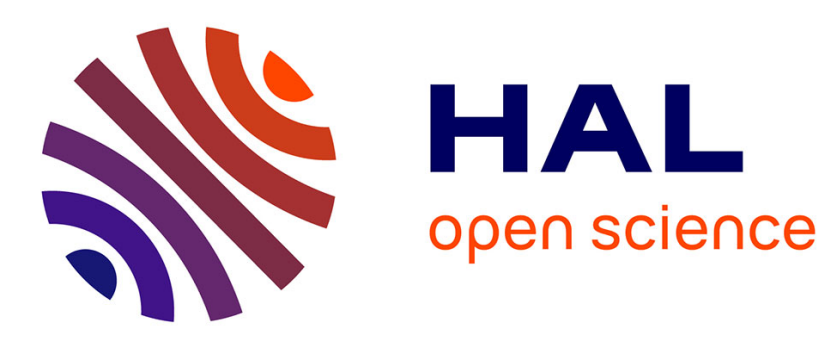

\title{
Blind Carrier Frequency Offset Estimation in Generalized Frequency Division Multiplexing
}

Ana Martinez, Maximilian Matthé, Marwa Chafii, Gerhard Fettweis

\section{To cite this version:}

Ana Martinez, Maximilian Matthé, Marwa Chafii, Gerhard Fettweis. Blind Carrier Frequency Offset Estimation in Generalized Frequency Division Multiplexing. IEEE ICNC 2019, Feb 2019, Honolulu, Hawaii, United States. hal-01965514

\section{HAL Id: hal-01965514 https://hal.science/hal-01965514}

Submitted on 26 Dec 2018

HAL is a multi-disciplinary open access archive for the deposit and dissemination of scientific research documents, whether they are published or not. The documents may come from teaching and research institutions in France or abroad, or from public or private research centers.
L'archive ouverte pluridisciplinaire HAL, est destinée au dépôt et à la diffusion de documents scientifiques de niveau recherche, publiés ou non, émanant des établissements d'enseignement et de recherche français ou étrangers, des laboratoires publics ou privés. 


\title{
Blind Carrier Frequency Offset Estimation in Generalized Frequency Division Multiplexing
}

\author{
Ana Belen Martinez*, Maximilian Matthé ${ }^{*}$, Marwa Chafii ${ }^{\ddagger}$, Gerhard Fettweis* \\ *Vodafone Chair Mobile Communications Systems, Technische Universität Dresden, Germany \\ \{ana-belen.martinez, fettweis\}eifn.et.tu-dresden.de \\ $\dagger^{\dagger}$ Barkhausen Institut gGmbH, Würzburger Str. 46, 01187 Dresden, Germany \\ maximilian.matthe@barkhauseninstitut.org \\ ${ }^{\ddagger}$ ENSEA, Cergy-Pontoise University, ETIS, CNRS, F-95000, Cergy, France \\ marwa.chafiidensea.fr
}

\begin{abstract}
In generalized frequency division multiplexing (GFDM), the self-generated inter-carrier interference can be exploited to estimate the carrier frequency offset (CFO). In this paper, the state-of-the-art algorithm for the blind estimation of the CFO in GFDM is evaluated in detail. For this purpose, the corresponding theoretical model under additive white Gaussian noise (AWGN) channel conditions is derived. Furthermore, an alternative approach to increase the accuracy of the estimation is proposed. Depending on the GFDM parameterization and channel conditions, the new method can significantly improve the mean square error (MSE) of the CFO without compromising the latency. Simulation results show that under AWGN channel conditions, a GFDM configuration with a low number of subsymbols can highly benefit from the proposed approach. In multipath fading channels, the MSE can be considerably reduced in systems with a moderate subcarrier spacing.
\end{abstract}

Index Terms-GFDM, blind, synchronization, carrier frequency offset (CFO), inter-carrier interference (ICI).

\section{INTRODUCTION}

With the definition of the scenarios envisioned for 5G [1], it became evident that the traditional orthogonal frequency division multiplexing (OFDM) could not be the technology of choice for the next generation of wireless communication systems. As a consequence, waveform design [2] has been widely investigated in the last years, and the corresponding research follows two different paths. The first one, based on OFDM, aims at improving its characteristics and solving its drawbacks. The second one focus on the design of alternative waveforms, as in the case of generalized frequency division multiplexing (GFDM) [3].

GFDM applies a circular filtering on a subcarrier basis and can transmit more than one data symbol per subcarrier, leading to a flexible non-orthogonal waveform. Due to its nonorthogonality, GFDM is subjected to inter-symbol interference (ISI) and inter-carrier interference (ICI) interference, fact that motivates the study of techniques to reduce the complexity required at the receiver [4] [5]. Since its introduction in 2009, numerous studies have shown its potential to meet the requirements of $5 \mathrm{G}[6]$.

Synchronization represents one of the most challenging issues at the receiver and plays an important role in the physical layer design. During the acquisition phase, the arrival time of the frame has to be accurately estimated. In addition, the carrier frequency offset (CFO), which appears due to the Doppler effects and the mismatch between the local oscillators at the transmitter and receiver, must be estimated and subsequently compensated to enable the proper working of the next modules of the receiver. In the framework of GFDM, data-aided (DA) as well as blind or non-data-aided (NDA) approaches have already been proposed.

The use of a training sequence, specifically designed according to the GFDM principles, either as a preamble [7], embedded in the data as a midamble [8] [9], or as a replacement for the cyclic prefix (CP) [10], is able to provide accurate time and fractional frequency synchronization. However, none of the mentioned approaches can provide a CFO estimation range higher than half of a subcarrier spacing.

Exploiting the periodicity provided by the CP in GFDM, a joint time and frequency offset estimator based on the maximum-likelihood (ML) principle was presented in [11]. The results showed comparable performance to those obtained for OFDM, concluding that the derived ML estimator is applicable to initial synchronization for GFDM as it is for OFDM. As a result, the CFO estimation range is still confined to half of a subcarrier spacing.

Besides the CP, the self-generated ICI in GFDM provides some periodic information which can be beneficial for synchronization. In [12], assuming a known time offset and making use of the intrinsic periodicity of the GFDM signal in frequency domain, a blind CFO estimator was introduced. Even though the acquisition range of the $\mathrm{CFO}$ extends to almost the whole frequency band, the high estimation error limits its use in practice.

The goal of this work is to improve the performance of the state-of-the-art blind CFO estimation approach in terms of the mean squared error (MSE) of the CFO estimation. For this purpose, a theoretical model of the metric used to estimate the CFO under additive white Gaussian noise (AWGN) channel conditions is derived, which allows for a better understanding of the method. The main factors that affect the performance of the approach are identified and an alternative method for the estimation of the CFO with higher accuracy is proposed.

The remainder of this paper is structured as follows: The 
blind CFO estimation approach is described in Section II. Section III presents the proposed method. Simulation results are discussed in Section IV. Finally, Section V concludes the paper.

\section{Blind CFO Estimation In FREQUEnCy Domain}

This section explains how the self-generated ICI in GFDM can be exploited for the estimation of the CFO and presents the approach from the mathematical point of view.

GFDM can be efficiently implemented in frequency domain using the sparsity of the pulse shaping filter in frequency domain [13]. In this case, the GFDM modulated signal can be expressed as:

$$
x[n]=\sum_{k \in \mathcal{K}} x_{k}[n],
$$

where $n=0,1, \ldots, N-1, \mathcal{K}$ represents the set of allocated subcarriers, and the signal $x_{k}[n]$ at the subcarrier $k$ is defined as:

$$
x_{k}[n]=\left[\sum_{m \in \mathcal{M}} d_{k, m} \delta[n-m K]\right] \underset{n, N}{\circledast} g[n] e^{j \frac{2 \pi k n}{K}},
$$

where $d_{k, m}$ stands for the complex-valued data symbol carried by the subcarrier $k$ and transmitted at the discrete time instant $m$. $N$ equals $K M$, with $K$ the number of subcarriers and $M$ the number of subsymbols. $\mathcal{M}$ refers to the set of active subsymbols, $g[n]$ denotes the pulse shaping filter with periodicity $N$ samples and $\circledast_{n, N}$ indicates the circular convolution with respect to the index $n$ and with periodicity of $N$ samples.

In frequency domain, the modulated signal at the subcarrier $k$ can be written as:

$$
\begin{aligned}
X_{k}[l] & =\operatorname{DFT}_{N}\left[\sum_{m \in \mathcal{M}} d_{k, m} \delta[n-m K]\right] \operatorname{DFT}_{N}[g[n]] \\
& \circledast \operatorname{DFT}_{N}\left[e^{j \frac{2 \pi k n}{K}}\right],
\end{aligned}
$$

where $\mathrm{DFT}_{N}[\cdot]$ stands for the $N$-point discrete Fourier transform (DFT). The term in the first squared brackets corresponds to a $K$-times upsampled version of the $M$ data symbols per subcarrier $k$. Therefore, its $N$-point DFT results in a $K$-times repetition of the $M$-point DFT of the set of data. This spectrum is multiplied by the frequency response of the pulse shaping filter, and finally, the product is convolved with the $N$-point DFT of the exponential term which indicates the position of corresponding subcarrier $k$.

In general, with a roll-off factor $\alpha$ of the pulse shaping filter $g[n]$ greater than zero, the bandwidth of $G[l]$ (where $G[l]=\mathrm{DFT}_{N}[g[n]]$ is the frequency domain response of the filter $g[n])$, extends more than the subcarrier spacing ( $M$ samples). As a consequence, $X_{k}[l]$ contains repeated information of the signal, weighted differently by the corresponding coefficients of the pulse shaping filter in frequency domain. This information appears with a periodicity of $M$ samples, over a length given by $M$ and the roll-off factor $\alpha$ as $V=2\lfloor\alpha M / 2\rfloor$ samples, where $\lfloor x\rfloor=\max \{n \in \mathbb{Z} \mid n \leq x\}$.

When the contribution of each subcarrier is added in frequency domain to build the modulated signal
$X[l]=\sum_{k \in \mathcal{K}} X_{k}[l]$, the periodic information from different subcarrier signals overlaps causing the characteristic selfgenerated ICI in GFDM.

GFDM can be configured to use CP and cyclic suffix (CS), which are added to the signal $x[n]$ to form a block. In general, $B$ blocks are concatenated to build the transmitted frame. At the receiver, after initial time synchronization, each individual block is extracted, as well as the CP and the CS, yielding, per block, the $N$-samples length signal $y[n]$ :

$$
y[n]=x[n] e^{j 2 \pi \epsilon n}+w[n],
$$

where $\epsilon$ accounts for the CFO normalized by the sampling frequency and $w[n]$ is AWGN with zero mean and variance $N_{0}$.

\section{A. Autocorrelation-Metric}

The main idea behind this approach is to exploit the repeated information of the signal for the estimation of the CFO. This intrinsic periodicity suggests an approach based on the autocorrelation of the received signal in frequency domain.

The $N$-point DFT of $y[n]$ can be formulated as:

$$
Y[l]=\sum_{k \in \mathcal{K}} \sum_{m \in \mathcal{M}} d_{k, m} G[l] e^{-j \frac{2 \pi K m l}{N}} \circledast I_{k}[l]+W[l],
$$

where

$$
\begin{aligned}
I_{k}[l] & =\operatorname{DFT}_{N}\left[e^{j\left(\frac{2 \pi k}{K}+2 \pi \epsilon\right) n}\right] \\
& =N \frac{\operatorname{sinc}[l-(k M+\epsilon N)]}{\operatorname{sinc}\left[\frac{1}{N}(l-(k M+\epsilon N))\right]} e^{j \pi \frac{N-1}{N}(l-(k M+\epsilon N))},
\end{aligned}
$$

and $\operatorname{sinc}[x]=\sin [\pi x] /[\pi x]$.

With

$$
P_{k, m}[l]=G[l] e^{-j 2 \pi \frac{K m l}{N}} \circledast I_{k}[l],
$$

the received signal in frequency domain can be finally expressed as:

$$
Y[l]=\sum_{k \in \mathcal{K}} \sum_{m \in \mathcal{M}} d_{k, m} P_{k, m}[l]+W[l] .
$$

The autocorrelation of $Y[l]$ at every frequency bin $l$ is calculated using a sliding window of $M$ samples and averaging over $V$ samples:

$$
\begin{aligned}
R_{Y}[l] & =\sum_{p=0}^{V-1} \mathbb{E}\left[Y^{*}\left[<l+p>_{N}\right] Y\left[<l+p+M>_{N}\right]\right] \\
& =\sum_{p=0}^{V-1}\left[\sum_{k \in \mathcal{K}} \sum_{m \in \mathcal{M}} \sum_{k^{\prime} \in \mathcal{K}} \sum_{m^{\prime} \in \mathcal{M}} \mathbb{E}\left[d_{k, m^{*}}^{*} d_{k^{\prime}, m^{\prime}}\right]\right. \\
& \left.\times P_{k, m}^{*}\left[<l+p>_{N}\right] P_{k^{\prime}, m^{\prime}}\left[<l+p+M>_{N}\right]\right] \\
& +\sum_{p=0}^{V-1}\left[\sum_{k \in \mathcal{K}} \sum_{m \in \mathcal{M}} \mathbb{E}\left[d_{k, m}^{*} W[l+p+M]\right]\right. \\
& \left.\times P_{k, m}^{*}\left[<l+p>_{N}\right]\right] \\
& +\sum_{p=0}^{V-1}\left[\sum_{k^{\prime} \in \mathcal{K}} \sum_{m^{\prime} \in \mathcal{M}} \mathbb{E}\left[d_{k^{\prime}, m^{\prime}} W^{*}[l+p]\right]\right.
\end{aligned}
$$




$$
\begin{aligned}
& \left.\times P_{k^{\prime}, m^{\prime}}\left[<l+p+M>_{N}\right]\right] \\
& +\sum_{p=0}^{V-1}\left[\mathbb{E}\left[W^{*}[l+p] W[l+p+M]\right]\right] .
\end{aligned}
$$

Under the assumption that the complex data symbols are independent and identically distributed (i.i.d.), and the data and the noise are independent, with $k=k^{\prime}, m=m^{\prime}$, $\sigma_{d}^{2}=\mathbf{E}\left[\left|d_{k, m}\right|^{2}\right]$ and $\mathbf{E}\left[d_{k, m}\right]=0$, the autocorrelation can be written as:

$$
R_{Y}[l]=\sigma_{d}^{2} \sum_{k \in \mathcal{K}} \sum_{m \in \mathcal{M}} \Lambda_{k, m}[l]+Z[l],
$$

where

$$
\Lambda_{k, m}[l]=\sum_{p=0}^{V-1} P_{k, m}^{*}\left[<l+p>_{N}\right] P_{k, m}\left[<l+p+M>_{N}\right],
$$

and $Z[l]$ accounts for the autocorrelation of the noise.

Fig. 1a shows the magnitude of the autocorrelation $\left|R_{Y}[l]\right|$ of a received signal $y[n]$ for different values of CFO. Information about the GFDM parameters used to generate the transmitted signal $x[n]$ can be found in Fig. 1. For the simulations, an AWGN channel with a signal-to-noise ratio (SNR) value of $20 \mathrm{~dB}$ has been considered, and 20 blocks have been averaged to obtain each individual $R_{Y}[l]$. Due to the periodicity of the signal, the autocorrelation exhibits one peak for each allocated subcarrier. The position of each maximum indicates the frequency shift respect to the corresponding subcarrier of the transmitted signal. If the CFO is an integer multiple of the frequency bin ${ }^{1}$, i.e. $(\epsilon=p / N$, with $p \in \mathbb{Z})$, all maxima of $R_{Y}[l]$ have, ideally, the same magnitude (red curve, $\mathrm{CFO}=7.0 / N)$. In this case, the term $I_{k}[l]$ in (6) reduces to a Kronecker delta function $\delta[l-(k M+\epsilon N)]$ and its convolution with $G[l]$ in (7) only shifts the position of $G[l]$ but does not modify its spectrum. As a result, $R_{Y}[l]$ is completely defined by the autocorrelation of $G[l]$ and the number of allocated subcarriers. For arbitrary values of $\mathrm{CFO}$ which are not integer multiples of $1 / N$, the convolution of the sampled Dirichlet function $I_{k}[l]$ with $G[l]$ does not correspond to a merely shifted version of $G[l]$. The worst case occurs for $\epsilon=(p \pm 0.5) / N$, when $I_{k}[l]$ does not have a global maximum but two local maxima of identical magnitude, leading to a high degradation of the metric.

\section{B. CFO-Metric}

In practice, with a low number of blocks, the local maxima of the calculated autocorrelation $R_{Y}[l]$ will have, depending on the data, different magnitude values and, as a consequence, $R_{Y}[l]$ cannot be considered a reliable metric for the CFO estimation. However, since the distance between two consecutive maxima of $R_{Y}[l]$ is $M$ samples, a more robust metric can be found by averaging the contribution of $M$-spaced $K_{o n}$ values of $R_{Y}[l]$ for every possible position of $l^{2}$. A global maximum

\footnotetext{
${ }^{1}$ Each frequency bin is considered to have a value of $1 / N$, where the bandwidth of the signal has been normalized to the unity for simplicity.

${ }^{2}$ Consecutive subcarrier allocation is considered here.
}

appears by full match of $K_{\text {on }}$ peaks, and its position indicates the frequency shift with respect to the first allocated subcarrier. The new metric, which in the following will be referred as CFO-Metric, is defined as:

$$
\Psi[l]=\frac{1}{K_{o n}} \sum_{k \in \mathcal{K}} R_{Y}\left[<l+k M>_{N}\right] .
$$

And the frequency offset can be calculated as:

$$
\hat{\epsilon}=\frac{\arg \max _{l \in \mathbb{Z}}\{\Psi[l]\}-l_{1}+V}{N},
$$

where $l_{1} \in \mathbb{Z}$ indicates the position of the first allocated subcarrier, and the factor $V$ compensates for the delay introduced by the autocorrelation. From (13), it becomes evident that the accuracy of the approach is limited to $1 / N$.

Fig. 1b illustrates the magnitude of the CFO-Metric $|\Psi[l]|$ obtained with the previously calculated $R_{Y}[l]$. For all evaluated cases, the global maximum is located at the same position, providing the same estimated $\mathrm{CFO}$ value. The fact that the global maximum obtained for CFO values of $6.7 / \mathrm{N}$ and $7.3 / N$ has a lower amplitude than for $7.0 / N$, as well as the asymmetry of their local maxima, indicates that the actual CFO is not an integer multiple of $1 / N$ and therefore, even under ideal channel conditions, the estimation has an implicit error, given as:

$$
\epsilon_{e}=\epsilon-\hat{\epsilon}
$$

It is worth mentioning that, under $\mathrm{CFO}$ values which are non integer multiples of $1 / N$, the CFO-Metric suffers a stronger degradation with decreasing number of subsymbols $M$. The ratio between the magnitude of the global maximum and the local maxima around it is reduced, leading to a high probability of wrong CFO estimations. Therefore, $M$ determines not only the accuracy of the approach, but also its robustness against arbitrary values of CFO.

Another parameter related to the robustness of the approach is the number of allocated subcarriers. If $K_{\text {on }} \leq K / 2$, the number of peaks of $\Psi[l]$ equals $2 K_{o n}-1$, and the global maximum can be clearly distinguished. But as the number of allocated subcarriers increases, due to the periodicity of $R_{Y}[l]$, the amplitude of the local maxima of $\Psi[l]$ increases, until the extreme case of full subcarrier allocation, where the CFOMetric consists of $K$ local maxima, and only the frequency shift within one subcarrier can be identified.

\section{PRoposed Method}

The performance of the presented approach depends on the parameters $K$ and $M$, which restrict the accuracy of the estimation to $1 / N$, as well as on the roll-off factor $\alpha$ of the pulse shaping filter, which together with $M$, determines the number of samples $V$ with duplicated information. Moreover, there is an uncertainty estimation range around the middle distance between two potential consecutive CFO values, and therefore, a high probability of error if the current $\mathrm{CFO}$ belongs to this region.

In the previous section it was shown, that the magnitude of the global maximum of $\Psi[l]$ depends on the value of the 


\begin{tabular}{|c|c|c|}
\hline $\begin{array}{l}\text { CFO }=6.7 / \mathrm{N} \text { Sim. } \\
-\cdot-\boldsymbol{C F O}=6.7 / \mathrm{N} \text { Theo }\end{array}$ & $\begin{array}{l}\text { CFO }=7.0 / \text { N Sim. } \\
-x_{n}-\mathrm{CFO}=7.0 / \mathrm{N} \text { Theo. }\end{array}$ & $\begin{array}{l}\text { CFO }=7.3 / \mathrm{N} \text { Sim. } \\
-* x \cdot \text { CFO }=7.3 / \mathrm{N} \text { Theo. }\end{array}$ \\
\hline
\end{tabular}

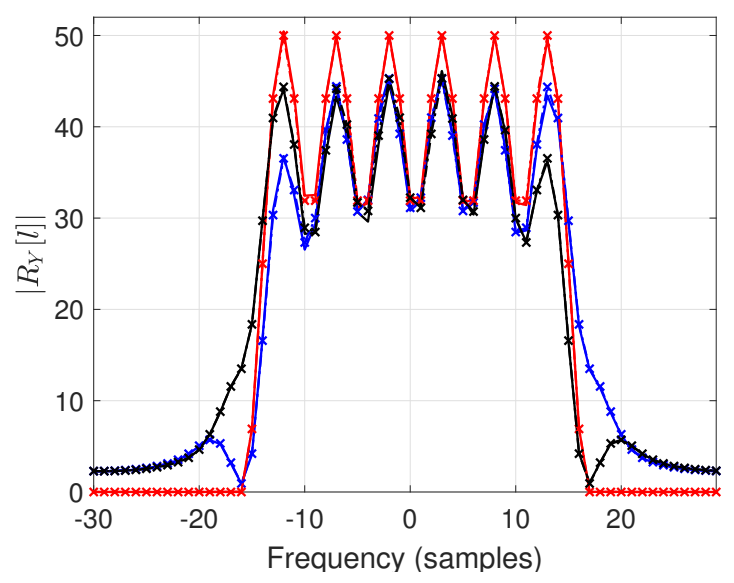

(a) Autocorrelation of received signal.

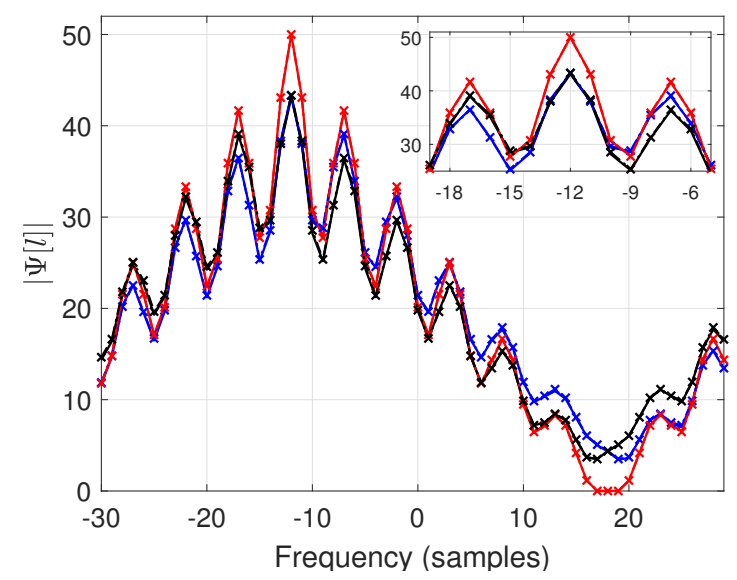

(b) CFO-Metric.

Fig. 1: a) Magnitude of autocorrelation $\left|R_{Y}[l]\right|$ and b) CFO-Metric $|\Psi[l]|$ in frequency domain. The GFDM parameters are $K=12, M=5$, and raised cosine with roll-off factor $\alpha=1$. The number of allocated subcarriers is $K_{o n}=6$ and the number of active subsymbols $M_{o n}=5$. For the evaluated CFO values $\epsilon, 6.7 / N, 7.0 / N$ and $7.3 / N$, the estimated CFO $\hat{\epsilon}$ is $7 / N$, leading to an estimation error $\epsilon_{e}$ of $-0.3 / N, 0 / N$ and $0.3 / N$, respectively. Simulation results are averaged over 10,000 realizations.

frequency offset $\epsilon$. This behavior is illustrated in Fig. 2 in more detail, where the magnitude of the global maximum of the CFO-Metric, obtained for different values of $\epsilon$ in the received signal, i.e. $\max _{l}|\Psi[l, \tilde{\epsilon}]|$, is represented as a function of $\epsilon$. The maxima of this function correspond to $\epsilon=p / N$, where $p \in \mathbb{Z}$, and the minima appear for $\epsilon=(p \pm 0.5) / N$.

\section{A. Maximization of the CFO-Metric}

The evolution of the magnitude of the maximum of $\Psi[l, \epsilon]$ as a function of $\epsilon$ indicates that the problem of low accuracy can be solved by finding the value of the frequency offset $\epsilon$ which, when compensated in the received signal, maximizes $\Psi[l, \epsilon]$. Let us define

$$
F(\tilde{\epsilon})=\max _{l} \Psi[l, \tilde{\epsilon}]
$$

as the function which contains the maximum value of $\Psi[l, \tilde{\epsilon}]$ for every possible value of $\tilde{\epsilon}$. The CFO value:

$$
\hat{\epsilon}=\underset{\tilde{\epsilon}}{\arg \max } F(\tilde{\epsilon})
$$

which maximizes the function $F(\tilde{\epsilon})$ corresponds to the fractional $\mathrm{CFO}, \hat{\epsilon}_{F}$, which allows for an enhancement of the accuracy. Moreover, the estimated $\hat{\epsilon}_{F}$ can be used to further improve the initial integer estimation. By compensating $\hat{\epsilon}_{F}$ in the received signal, an improved CFO-Metric with reduced uncertainty can be obtained. Therefore, a more accurate estimation of the integer CFO, $\hat{\epsilon}_{I}$, given by the global maximum of $\Psi\left[l, \hat{\epsilon}_{F}\right]$, can be achieved. Hence, the new CFO estimation $\hat{\epsilon}$ is given as the sum of two terms:

$$
\hat{\epsilon}=\hat{\epsilon}_{I}+\hat{\epsilon}_{F} \text {. }
$$

Well-known optimization algorithms can be used to find the solution to this problem with high accuracy.

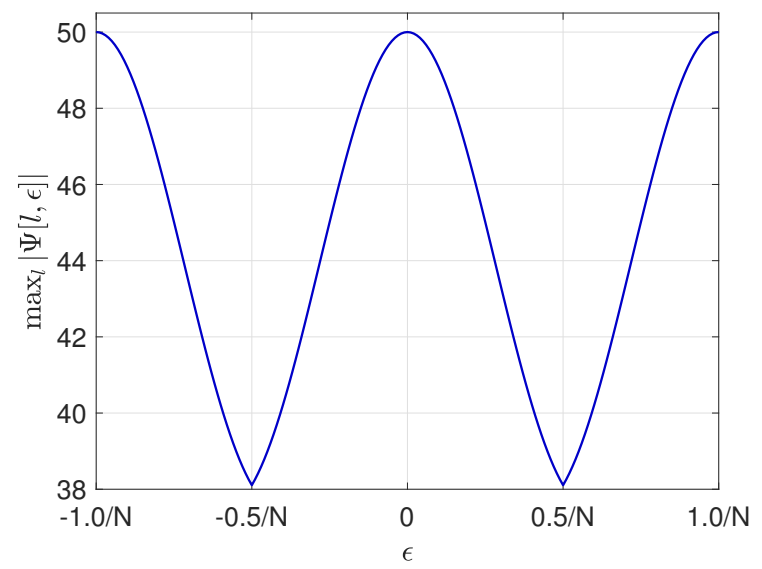

Fig. 2: Evolution of the magnitude of the global maximum of the CFO-Metric $|\Psi[l, \epsilon]|$ as a function of $\epsilon$.

\section{B. Manual Search of the CFO}

This work proposes a manual search of the new CFO. The necessary steps are summarized as follows:

1) Definition of the observation space of trial CFO values. The range limits are defined to cover all values of interest, i.e. $[-0.5 / N,+0.5 / N]$, so that the range span, $\Delta R$, corresponds to the frequency bin spacing $1 / N$.

2) Definition of the resolution between trial $\mathrm{CFO}$ values. With $T$ being the number of trial CFO values at each side of the middle of the observation space, the resolution corresponds to $\Delta \tilde{\epsilon}=\Delta R /(2 T+1)$, and the 
value of each trial CFO $\tilde{\epsilon}_{i}$ is given by: $\tilde{\epsilon}_{i}=i \Delta \tilde{\epsilon}$, where $i=-T,-T+1, \ldots, T-1, T$.

3) For every trial $\mathrm{CFO} \tilde{\epsilon}_{i}$ :

a) Compensation of the trial $\mathrm{CFO}$ value $\tilde{\epsilon}_{i}$ in the received signal $y_{i}\left[n, \tilde{\epsilon}_{i}\right]$.

b) Calculation of the corresponding CFO-Metric $\Psi_{i}\left[l, \tilde{\epsilon}_{i}\right]$ and its maximum $\tilde{F}\left[\tilde{\epsilon}_{i}\right]=\max _{l} \Psi_{i}\left[l, \tilde{\epsilon}_{i}\right]$.

4) Selection of the maximum of $\tilde{F}\left[\tilde{\epsilon}_{i}\right]$. The fractional CFO estimation $\hat{\epsilon}_{F}$ is obtained as $\hat{\epsilon}_{F}=\arg \max _{\tilde{\epsilon}_{i}} \tilde{F}\left[\tilde{\epsilon}_{i}\right]$. The maximum of the previously calculated $\Psi_{i}\left[l, \hat{\epsilon}_{F}\right]$ provides a new integer CFO estimation, $\hat{\epsilon}_{I}$, as described in (13).

It is worth mentioning that the calculation of each individual CFO-Metric, $\Psi_{i}\left[l, \tilde{\epsilon}_{i}\right]$, does not require information from previous steps and therefore, all of them can be computed in parallel, resulting in an approach with an overall higher computational complexity that the conventional one, but without additional latency. Besides, the complexity can be adjusted with a suitable definition of the resolution $\Delta \tilde{\epsilon}$.

\section{Simulation Results}

In this section, the performance of the proposed approach is evaluated and compared to the existing one. Simulations have been performed under AWGN and frequency selective fading channel conditions. In the latter case, an exponential power delay profile with a maximum delay length of $N_{c p} / 2-1$ samples is chosen. The ratio of the first tap to the last one is set to $20 \mathrm{~dB}$. Different values of the parameters $K$ and $M$ are considered to observe their influence on the results. Table I contains the sets of parameters used for this evaluation. In all cases, a raised cosine filter with roll-off factor $\alpha=1$ is selected as a pulse shaping filter, the number of allocated subcarriers is half of the total number of subcarriers, the length of the CP is set to $N / 4$, and the number of blocks to average the results of the autocorrelation is equal to 20 . The signal bandwidth is in every case the same and normalized to unity. The CFO is uniformly distributed between $-K / 2+1$ and $K / 2-1$, and can take any arbitrary value in this range, i.e. is not restricted to integer multiples of $1 / N$. For the manual search of the CFO, a resolution step $\Delta \tilde{\epsilon}=0.1 / N$ is chosen, with $T=5$.

TABLE I: GFDM parameterization.

\begin{tabular}{ccccccc}
\hline & $K$ & $K_{\text {on }}$ & $M$ & $M_{\text {on }}$ & $N$ & $N_{c p}$ \\
\hline set 1 & 24 & 12 & 3 & 3 & 72 & 18 \\
set 2 & 8 & 4 & 9 & 9 & 72 & 18 \\
set 3 & 24 & 12 & 9 & 9 & 216 & 54 \\
\hline
\end{tabular}

In order to evaluate the performance of each approach, the MSE is used, defined as:

$$
\operatorname{MSE}(\epsilon)=\mathbb{E}\left[(\epsilon-\hat{\epsilon})^{2}\right] .
$$

\section{A. MSE Under AWGN Channel Conditions}

Fig. 3a shows the MSE of the CFO obtained for all parameter sets under AWGN channel conditions, for SNR values from 0 to $20 \mathrm{~dB}$. The MSE provided by the initial approach indicates the dependence of the results on the parameters $K$ and $M$. With the first set of parameters, the low number of subsymbols $M$ is responsible for the higher degradation of the CFO-Metric with respect to the other two cases, and therefore, a higher MSE than in the second and third sets is achieved.

Sets two and three present the same accuracy with respect to the subcarrier spacing $1 / M$, and differ only in the number of subcarriers $K$. Accordingly, the difference between their corresponding MSE curves reside mainly in the ratio between the mentioned number of subcarriers. A normalized MSE should show similar performances.

The effect of the proposed approach on the MSE is especially remarkable in the first case, where the compensation of the estimated fractional CFO provides a more reliable integer CFO estimation. The incorporation of the fractional CFO allows for an even further MSE reduction. In the low SNR region, the new approach achieves a considerable improvement of the MSE in all cases. With a high number of subsymbols (second and third cases), the MSE floor observed at high SNR values is determined mainly by the number of blocks used for the calculation of the metrics, and the influence of the proposed approach is strongly reduced.

\section{B. MSE Under Multipath Fading Channel Conditions}

Under multipath fading channel conditions, the frequency selectivity affects the frequency bins which are used to calculate the autocorrelation differently. As a consequence, the autocorrelation degrades, leading to an increase of the MSE. Fig. $3 \mathrm{~b}$ depicts the MSE of the CFO obtained for all considered sets.

The improved integer CFO estimation is able to reduce the MSE in all considered sets, especially in the first one, due to the remarkable improvement of the CFO-Metric. The initial estimation obtained for the second and third sets reflects the effect of the number of subcarriers in the accuracy. However, the improvement introduced by the new approach is more evident in the third set. The higher number of subcarriers and therefore, the lower subcarrier spacing, under the assumption of identical signal bandwidth, has a positive impact on the performance of the proposed method. Due to the frequency variability, the fractional $\mathrm{CFO}$ does not introduce a visible effect on the MSE.

\section{Estimation Uncertainty}

In order to illustrate the effect of the estimation uncertainty on the MSE and the improvement achieved by the proposed approach, simulations under ideal channel conditions have been performed. The $\mathrm{CFO}$ evaluation range extends from $-0.5 / N$ to $0.5 / N$ and the resolution step $\Delta \tilde{\epsilon}$ has been set to $0.025 / N$. The GFDM parameters correspond to the first set, where the difference among the different CFO estimations at high SNRs is particularly significant. Fig. 4 shows the MSE of the CFO obtained with the conventional and the new approach. As expected, due to the strong degradation of the CFO-Metric, the highest values of MSE appear in the areas around $\pm 0.5 / N$. It is in these areas where the new approach achieves a remarkable improvement of the integer 


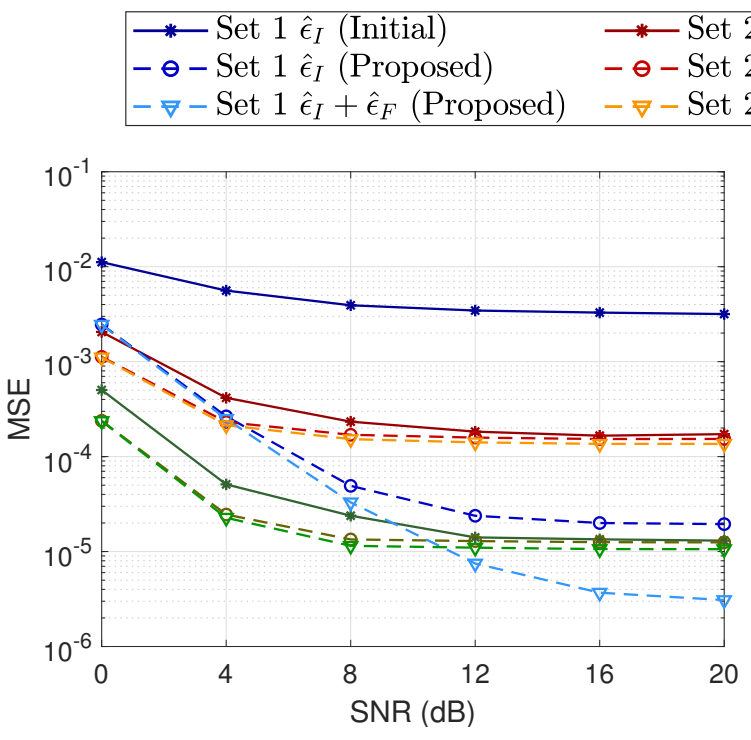

(a) AWGN channel.
—- Set $3 \hat{\epsilon}_{I}$ (Initial)

$-\ominus-\operatorname{Set} 3 \hat{\epsilon}_{I}$ (Proposed)

$-\nabla-\operatorname{Set} 3 \hat{\epsilon}_{I}+\hat{\epsilon}_{F}$ (Proposed)

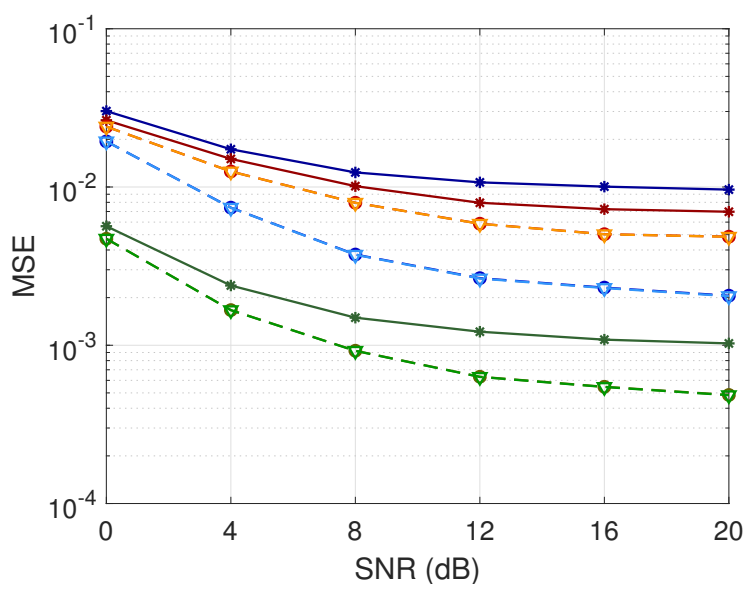

(b) Frequency selective fading channel.

Fig. 3: MSE of CFO obtained with initial and proposed approaches for different GFDM configurations and channel conditions.

CFO estimation and a high reduction of the corresponding MSE. It can be seen that the MSE obtained with the new integer CFO estimation is close to the theoretical one. With the incorporation of the fractional CFO, an almost constant MSE in the whole evaluation range is achieved.

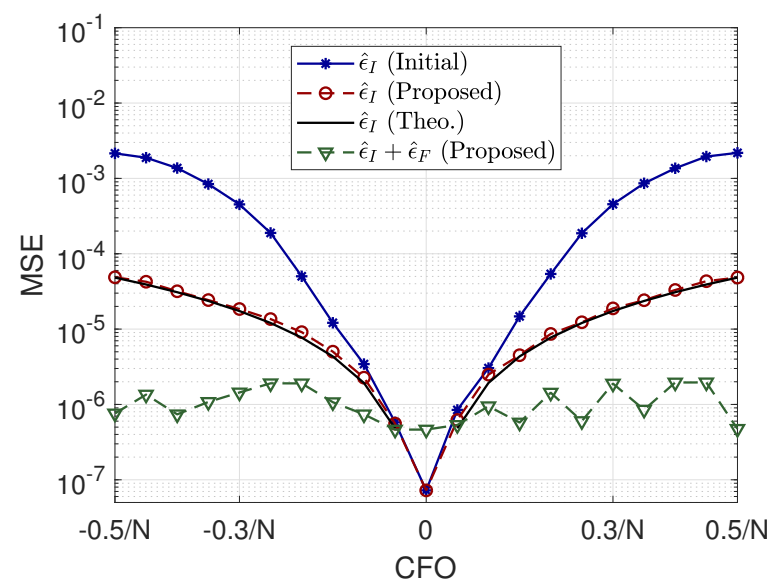

Fig. 4: MSE of CFO obtained for the first set of GFDM parameters under ideal channel conditions.

\section{Conclusions}

In this paper, the use of the self-generated ICI in GFDM for the estimation of the CFO has been thoroughly examined. With the help of the theoretical model derived in this work, the influence of the GFDM parameters on the accuracy of the estimation and on the robustness of the CFO-Metric has been addressed. Besides, this model has enabled to identify the dependence of the CFO on the CFO-Metric.

Once the relationship between the CFO-Metric and the actual CFO of the system has been established, a new approach to improve the MSE of the estimation has been proposed. Its performance has been evaluated and compared to the one obtained with the existing approach. It has been shown that, depending on the GFDM parameterization and channel conditions, the new method can achieve a significant reduction of the MSE.

In AWGN channels, the new approach can improve the MSE in the low SNR operating area for all evaluated systems, while for high SNR values, the effect of the proposed algorithm is reduced as the number of subsymbols $M$ increases.

Under frequency fading channel conditions, all evaluated cases can benefit from the new approach. However, it has to be considered, that the performance degradation of the metric, caused by the frequency fading, should remain within acceptable limits for the method to work properly.

The main advantage of the blind CFO estimation approach is that it offers the possibility to estimate the CFO over a high frequency range without compromising the spectral efficiency. At the cost of a higher, but adjustable computational complexity, the proposed algorithm improves the CFO estimation performance in terms of the MSE. By means of parallel processing, the latency required to provide the estimation does not need to increase. However, it is non-negligible and has to be taken into account in practical implementations. Therefore, streaming transmissions appear as potential applications which can benefit from the proposed approach. After initial time synchronization, the CFO estimation based on a fixed number of blocks can be performed as described in this work. Subsequently, a new estimation can be done following a sliding window principle, where the first arrived block is no longer considered in the calculation, and a new one is incorporated to the algorithm. This process can be carried out continuously over the whole frame for tracking purposes. 


\section{ACKNOWLEDGEMENT}

The research leading to these results has received funding from the European Union's Horizon 2020 Research and Innovation Programme under the grant agreement No. 732174 (ORCA). The computations were performed on a Bull Cluster at the Center for Information Services and High Performance Computing (ZIH) at TU Dresden.

\section{REFERENCES}

[1] A. Osseiran, F. Boccardi, V. Braun, K. Kusume, P. Marsch, M. Maternia, O. Queseth, M. Schellmann, H. Schotten, H. Taoka, H. Tullberg, M. A. Uusitalo, B. Timus, and M. Fallgren, "Scenarios for 5G mobile and wireless communications: the vision of the METIS project," IEEE Communications Magazine, vol. 52, no. 5, pp. 26-35, May 2014.

[2] A. Farhang, N. Marchetti, F. Figueiredo, and J. P. Miranda, "Massive mimo and waveform design for 5 th generation wireless communication systems," in $5 \mathrm{~g}$ for Ubiquitous Connectivity (5gu), 2014 1st International Conference on. IEEE, 2014, pp. 70-75.

[3] G. Fettweis, M. Krondorf, and S. Bittner, "Gfdm-generalized frequency division multiplexing," in Vehicular Technology Conference, 2009. VTC Spring 2009. IEEE 69th. IEEE, 2009, pp. 1-4.

[4] M. Matthe, I. Gaspar, D. Zhang, and G. Fettweis, "Reduced complexity calculation of lmmse filter coefficients for gfdm," in Vehicular Technology Conference (VTC Fall), 2015 IEEE 82nd. IEEE, 2015, pp. 1-2.

[5] A. Farhang, N. Marchetti, and L. E. Doyle, "Low complexity gfdm receiver design: A new approach," in Communications (ICC), 2015 IEEE International Conference on. IEEE, 2015, pp. 4775-4780.

[6] N. Michailow, M. Matthé, I. S. Gaspar, A. N. Caldevilla, L. L. Mendes, A. Festag, and G. Fettweis, "Generalized frequency division multiplexing for 5th generation cellular networks," IEEE Transactions on Communications, vol. 62, no. 9, pp. 3045-3061, 2014.

[7] I. S. Gaspar, L. L. Mendes, N. Michailow, and G. Fettweis, "A synchronization technique for generalized frequency division multiplexing," EURASIP Journal on Advances in Signal Processing, vol. 2014, no. 1, p. 67,2014

[8] I. Gaspar and G. Fettweis, "An embedded midamble synchronization approach for generalized frequency division multiplexing," in Global Communications Conference (GLOBECOM), 2015 IEEE. IEEE, 2015, pp. $1-5$.

[9] K. Lee, M. Kang, E.-R. Jeong, D.-J. Park, and Y. H. Lee, "Use of training subcarriers for synchronization in low latency uplink communication with gfdm," in Signal Processing Advances in Wireless Communications (SPAWC), 2016 IEEE 17th International Workshop on. IEEE, 2016, pp. 1-6.

[10] I. Gaspar, A. Festag, and G. Fettweis, "Synchronization using a pseudocircular preamble for generalized frequency division multiplexing in vehicular communication," in Vehicular Technology Conference (VTC Fall), 2015 IEEE 82nd. IEEE, 2015, pp. 1-5.

[11] P.-S. Wang and D. W. Lin, "Maximum-likelihood blind synchronization for gfdm systems," IEEE Signal Processing Letters, vol. 23, no. 6, pp. 790-794, 2016.

[12] T. Kadur, I. Gaspar, N. Michailow, and G. Fettweis, "Non-data aided frequency synchronization exploiting ici in non-orthogonal systems," in Vehicular Technology Conference (VTC Fall), 2014 IEEE 80th. IEEE, 2014, pp. 1-5.

[13] I. Gaspar, N. Michailow, A. Navarro, E. Ohlmer, S. Krone, and G. Fettweis, "Low complexity gfdm receiver based on sparse frequency domain processing," in 2013 IEEE 77th Vehicular Technology Conference (VTC Spring), June 2013, pp. 1-6. 